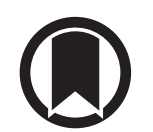

CrossMark

\title{
Relative and absolute lung function change in a general population aged $60-102$ years
}

\author{
Johannes Luoto (i]', Mats Pihlsgård ${ }^{1}$, Per Wollmer ${ }^{2}$ and Sölve Elmståhl ${ }^{1}$ \\ Affiliations: ${ }^{1}$ Dept of Clinical Sciences in Malmö, Division of Geriatric Medicine, Skåne University Hospital, \\ Lund University, Malmö, Sweden. ${ }^{2}$ Clinical Physiology and Nuclear Medicine Unit, Dept of Translational \\ Medicine, Skåne University Hospital, Lund University, Malmö Sweden.
}

Correspondence: Johannes Luoto, Dept of Clinical Sciences in Malmö, Division of Geriatric Medicine, Jan Waldenströms gata 35, SE-205 02, Malmö, Sweden. E-mail: johannes.luotodamed.lu.se

@ERSpublications

Female sex, ageing and inflammation significantly increased relative but not absolute lung function decline in new Swedish study. NHANES III may offer better lung function reference equations than GLI for elderly: findings from a large cohort aged 65-102. http://ow.ly/Szca30n3JDo

Cite this article as: Luoto J, Pihlsgård M, Wollmer P, et al. Relative and absolute lung function change in a general population aged 60-102 years. Eur Respir J 2019; 53: 1701812 [https://doi.org/10.1183/ 13993003.01812-2017].

ABSTRACT Data on longitudinal lung function change in the elderly are scarce. Uncertainty remains about whether to use absolute or relative change and how it relates to subject demographics.

We studied absolute and relative forced expiratory volume in $1 \mathrm{~s}$ (FEV1) and forced vital capacity (FVC) change in a population-based geriatric sample using a repeated measurements model adjusted for age, sex, smoking habits, heart failure, hypertension, diabetes, coronary heart disease, educational level, occupation, alcohol consumption, C-reactive protein (CRP) and body mass index. 3736 participants aged 60-102 years completed between one and five spirometries during 13.5 years of follow-up. Lung volumes, FEV1 quotient (Q) and Global Lung Initiative (GLI)-2012 and National Health and Nutrition Examination Survey (NHANES) III z-scores were presented from 6932 spirometries.

Adjusted absolute change per year (95\% CI) was -51.7 (-63.7--39.9) $\mathrm{mL}$ for FEV 1 and $-56.2(-73.6-$ $-38.8) \mathrm{mL}$ for FVC. Adjusted relative change per year was $-2.97(-3.53--2.40) \%$ for FEV1 and -2.46 $(-3.07--1.85) \%$ for FVC. Risk factors for increased relative FVC and FEV1 decline were female sex, higher age, current smoking habits, elevated CRP (nonsignificant for FEV1, p=0.057) and low educational level. For increased absolute decline the risk factors were male sex and being a current smoker for FEV1 and low education for FVC.

Relative but not absolute change correlated significantly with clinically relevant markers of functional status and may be superior to absolute change in risk factor analysis. Cross-sectional reduction in terms of FEV 1 Q was $\sim 1$ unit per 10 years for both sexes. Proportions of subjects with results below lower limit of normal using NHANES III were close to anticipated, but were two to four times higher than expected using GLI-2012.

This article has supplementary material available from erj.ersjournals.com

Received: May 242017 | Accepted after revision: Dec 142018

Copyright OERS 2019. This version is distributed under the terms of the Creative Commons Attribution Licence 4.0. 


\section{Introduction}

Although forced expiratory volume in $1 \mathrm{~s}\left(\mathrm{FEV}_{1}\right)$ is an independent predictor of death, and morbidity in general populations [1] and severity of airflow limitation in chronic obstructive pulmonary disease (COPD) is determined by it $[2,3]$, recent studies covering change of FEV1 in general populations are scarce and there are no previous studies reporting findings for subjects aged $>90$ years [4]. Furthermore, it is unclear whether the rate of lung function decline increases with high age or whether it remains unaffected by ageing [1,4-7]. In addition, the importance of other risk factors besides smoking is becoming increasingly clear, with female sex $[1,4,8]$ and socioeconomic status [9] linked to poor lung function and increased lung function decline. C-reactive protein (CRP), a biomarker for inflammation, has been linked to increased lung function decline in an elderly population [4]. Occupational category as well as exposure to dusts, gases and fumes have been linked to increased lung function decline and respiratory symptoms in younger general populations [10-12], but the long-term effects of occupation have not been studied in populations that are past retirement age. In light of this, more studies on the effects of sex, socioeconomic status and occupational exposure on lung function have been requested by the European Respiratory Society [13]. There is no consensus as to what is the appropriate measure of change. Modelling change relatively has been proposed as an alternative to an absolute model of change [14-16].

We used 13-year follow-up data of subjects aged 60-102 years from the general population cohort study Good Aging in Skåne (GÅS) in order to assess the rates of absolute and relative change in FEV1 and forced vital capacity (FVC) and how they are affected by the aforementioned characteristics [17].

We hypothesised that the rate of absolute and relative lung function decline is increased with higher age, active smoking habits, low educational level, high-risk occupations, high CRP and with female sex. As a secondary aim, we examined the influence of comorbidities previously linked to poor lung function or increased lung function decline.

\section{Methods}

\section{Study design and participants}

This study is part of the ongoing longitudinal GÅS study, which in turn is part of the Swedish National Study on Aging and Care (SNAC). These have been described previously [17-19]. In short, GÅS includes subjects randomly selected from the Swedish municipality register of the rural and urban general population of the southern Swedish province of Skåne. Two separate random selections of participants were made: cohort I, aged 60-95 years at baseline, with baseline examinations in 2001; and cohort II, aged 60-81 years at baseline, with baseline examinations in 2006. The overall response rate was $63 \%$. The study designs for the two cohorts were identical, with subjects aged $<80$ years invited for follow-up examination every six years, and subjects aged $\geqslant 80$ years every 3 years. To date, three examinations involving cohort I participants aged $<80$ years are completed. The corresponding numbers for cohort I participants aged $\geqslant 80$ years, cohort II participants aged $<80$ years and cohort II participants $\geqslant 80$ years are five, two and three examinations, respectively.

All examinations were performed according to the same protocol with the same data collected on each occasion. Assessments consisted of questionnaires, physical examinations, spirometry and blood sampling as detailed later. The assessments were performed on one, or in some cases two consecutive days at the research clinic or at the subject's home or in sheltered housing. Making home calls was a deliberate strategy to reduce selection bias due to morbidity and frailty.

The inclusion criterion was at least one acceptable spirometry according to American Thoracic Society (ATS) criteria [20].

\section{Definition of variables and subgroups}

Subjects filled out a self-report questionnaire regarding their history of smoking habits, alcohol consumption and medical history. Subjects uncertain of their medical history had their medical records reviewed by a physician. A nurse recorded height, weight, timed get-up-and-go test (TUG) and time required to walk $15 \mathrm{~m}$ at subjects' maximum speed. TUG was defined as time required to getting up from a straight-backed armchair, walking $3 \mathrm{~m}$ and returning to a seated position. Shoes and walking aids were allowed. Maximum handgrip strength was measured using an electronic gauge (Grippit; AB Detektor, Goteborg, Sweden) [21]. Smoking habits were categorised as current smokers, ex-smokers or never-smokers. We used the attained level of education as a marker of socioeconomic status. Educational level was categorised as low (did not complete elementary school or equivalent), medium (completed elementary school) or high ( $\geqslant 1$ year of post-elementary school education). Subjects were asked how many times per month they consume alcohol and how many glasses they consumed on a typical occasion; the product of these numbers was used as an approximation of monthly alcohol consumption measured in glasses per month. Occupations were coded according to the Swedish standard for occupational 
classification (SSYK 96). Three out of the 10 SSYK 96 main categories were identified as potential risk occupations. These categories were "farming, forestry and gardening", "construction and manufacturing" and "unqualified work". The first two due to possible exposure to dusts and fumes. Unqualified work was identified as a potential risk occupational category by representing low socioeconomic status. The remaining categories (managerial occupations, theoretical specialty competency, shorter college education, office work, customer service, nursing and sales, process and machine operators and military) were merged into a reference category. In order to identify cognitive impairment, a Mini Mental State Examination (MMSE) was conducted [22].

\section{Spirometry}

Five specially trained and highly experienced nurses performed the spirometries. The tests were performed using the Vitalograph model 2120 spirometer using Spirotrac IV software (Vitalograph, Buckingham, UK). The spirometry, including daily calibration, was performed according to ATS guidelines [20] at all examinations with the exception that a bronchodilator was not administered at baseline for cohort I. At all other examinations subjects received $1.0 \mathrm{mg}$ of $\beta_{2}$-receptor agonist terbutaline $10 \mathrm{~min}$ prior to the spirometry. In addition, only one acceptable manoeuvre was required for inclusion in the study sample. The motivation was to avoid introducing selection bias among the very old, who may not be able to perform multiple manoeuvres [23]. The Spirotrac software offers built in ATS test quality criteria with automatic feedback to the technician as a guidance when interpreting the quality of the spirometry. Up to a maximum of eight consecutive breathing manoeuvres were performed with the goal of completing at least three acceptable curves. Reproducibility criteria was used as an indication of whether more manoeuvres were necessary and not a basis for exclusion of subjects [20]. The same spirometer was used at all examinations, including when the test was performed away from the research clinic. FEV1 and FVC were analysed.

\section{Statistical analysis}

For each participant, we identified examinations with nonmissing FEV1 and FVC measurements that were 5.5-6.5 years apart. With a follow-up time of 13 years this means that each subject may contribute with one or two separate trajectories. The absolute outcome was the change in $\mathrm{mL}$ per year (calculated by subtracting the initial measurement from the follow-up measurement and dividing the result by the time between examinations). The relative outcome was constructed in the same way using logarithms of lung volumes rather than the volumes themselves. The multivariate models of change were modelled in repeated measurement models adjusted for sex [1, 4], smoking habits [7], heart failure [24], hypertension [25], diabetes [25], coronary heart disease [6], socioeconomic status [1, 4, 6], occupation [10-12], alcohol consumption [6], CRP [4, 26] and body mass index (BMI) [27, 28] (at the initial examination). The covariates included in the statistical analyses were all chosen a priori, i.e. no form of data-driven covariate selection process was applied. Crude estimates of relative change per year and corresponding least squares means for an ideal population were computed in order to facilitate the interpretation of results; further details can be found in the supplementary material. If covariate information was missing but available at an earlier or later examination, we used these values in order to reduce the number of excluded subjects (if information was available at both an earlier and later examination we used data from the earlier one). Additional analyses to estimate the significances of age-CRP interaction and sex-smoking interaction were conducted (with interaction terms added to the full model). To analyse which factors increased the risk of being excluded from the analyses, we conducted logistic regressions adjusted for the factors of interest (one at a time), age and sex with "participation with less than two spirometries" as outcome. The 2012 Global Lungs Initiative (GLI) and National Health and Nutrition Examination Survey (NHANES) III reference equations were used to calculate z-scores [29, 30]. The GLI-2012 SAS macro version 2 was used to calculate the GLI z-scores [31]. In analysis using z-scores, GLI z-scores were used unless otherwise stated. For subjects aged $>95$ years GLI equations were truncated by setting age to 95 years. To illustrate the relevance of functional status as a marker of frailty and its association with poorer lung function, these covariates (walking speed, TUG and grip strength) were added separately to the full model. In addition, they were analysed in the total population for estimation of effects on FEV1 and FVC GLI z-scores. FEV 1 was also expressed as FEV1 quotient (Q), the number of remaining turnovers for the estimated lowest sex-specific survivable FEV1 [32]. Trajectories with bronchodilation at first spirometry and those without were analysed separately to estimate whether they differed in outcome and significance pattern of risk factors. Since there was no a priori suspicion of disease the lower limit of normal (LLN) was defined as a Z-score $<-1.96$ [33], which is the lower 2.5th percentile. Further details regarding the statistical analyses can be found in the supplementary material. 
Statistical software

All analyses and figures were performed using SAS 9.3 (SAS, Cary, NC, USA) and RStudio (version 0.99.482; RStudio, Boston, MA, USA)

Ethics

The study was approved by the regional ethics committee of Lund University (2002; registration number LU 744-00) and all participants or carers provided written consent.

\section{Results}

Participants

Table 1 presents subject characteristics according to level of participation. Out of the 7611 invited, 57\% were female and mean age was 72 years. In total 6932 spirometries were performed. After invalid values were removed, $6837 \mathrm{FEV} 1$ and $6874 \mathrm{FVC}$ manoeuvres remained. The number of longitudinal trajectories are displayed in tables 3, 4, S1 and S2. Age and smoking habit specific FEV1, FVC, FEV1/FVC ratio and FEV1Q for the total population are presented in table 2 together with GLI-2012 z-scores. NHANES III $\mathrm{z}$-scores are available in the supplementary material. The number of longitudinal trajectories are displayed in tables 3, 4, S1 and S2. Across the study period the youngest participant was aged 60 years and the oldest was aged 102 years at the time of examination. At baseline, $4.5 \%$ of subjects who completed at least two spirometry visits had a FEV1/FVC ratio below the GLI-2012 LLN. The proportion of z-scores below the GLI-2012 LLN for FEV1 and FVC were $8.2 \%$ and 9.9\%, respectively. The proportions for NHANES Z-scores below the LLN were $2.1 \%$ for the FEV1/FVC ratio, $1.9 \%$ for FEV 1 and $1.8 \%$ for FVC. Among 387 never-smokers the proportions of GLI-2012 z-scores below the LLN were 3.9\% for the FEV1/FVC ratio,

TABLE 1 Baseline characteristics according to study participation

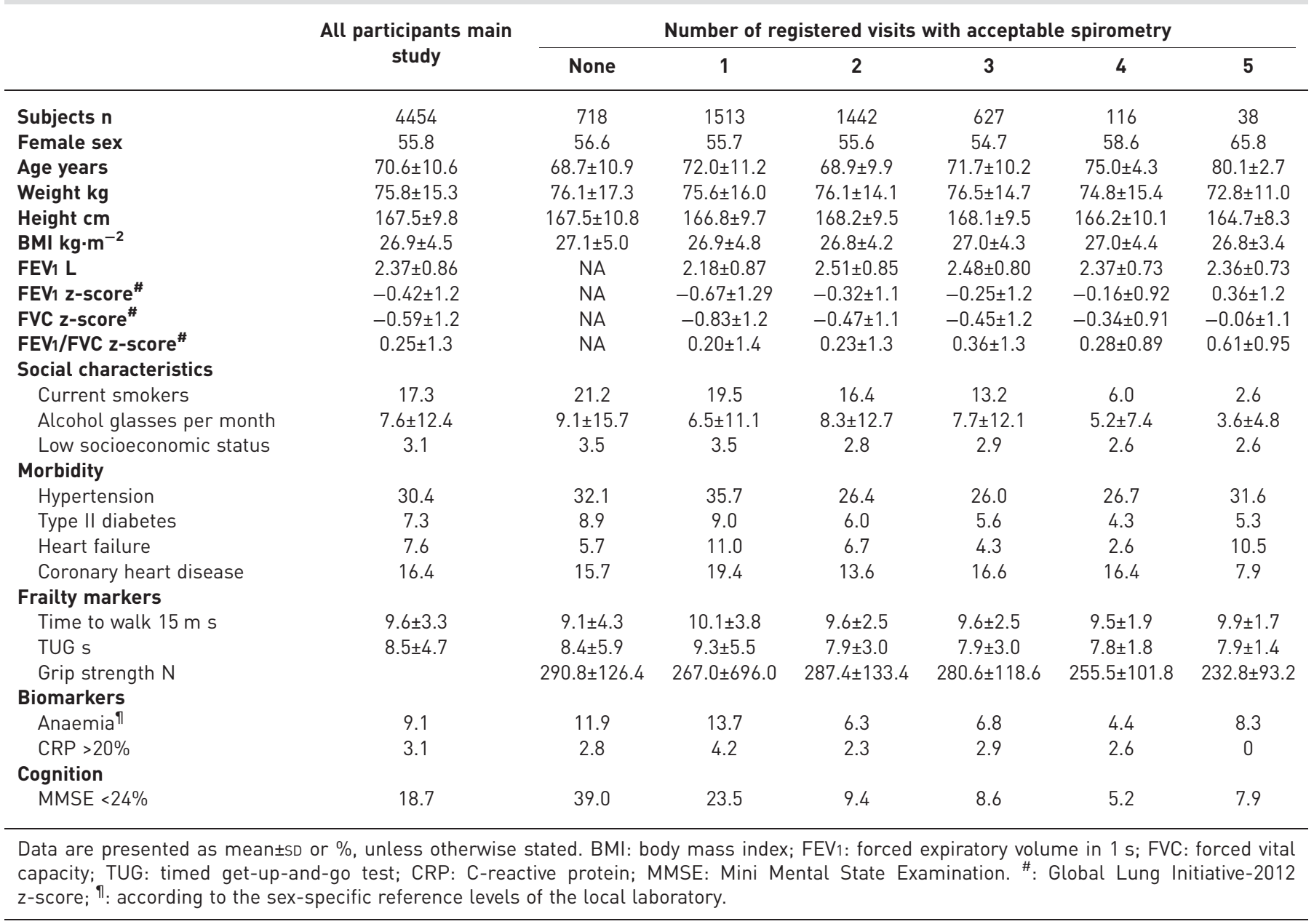


TABLE 2 Lung volumes, forced expiratory volume in 1 s quotient (FEV1Q) and Global Lung Initiative (GLI)-2012 z-scores for ages 60-102 years

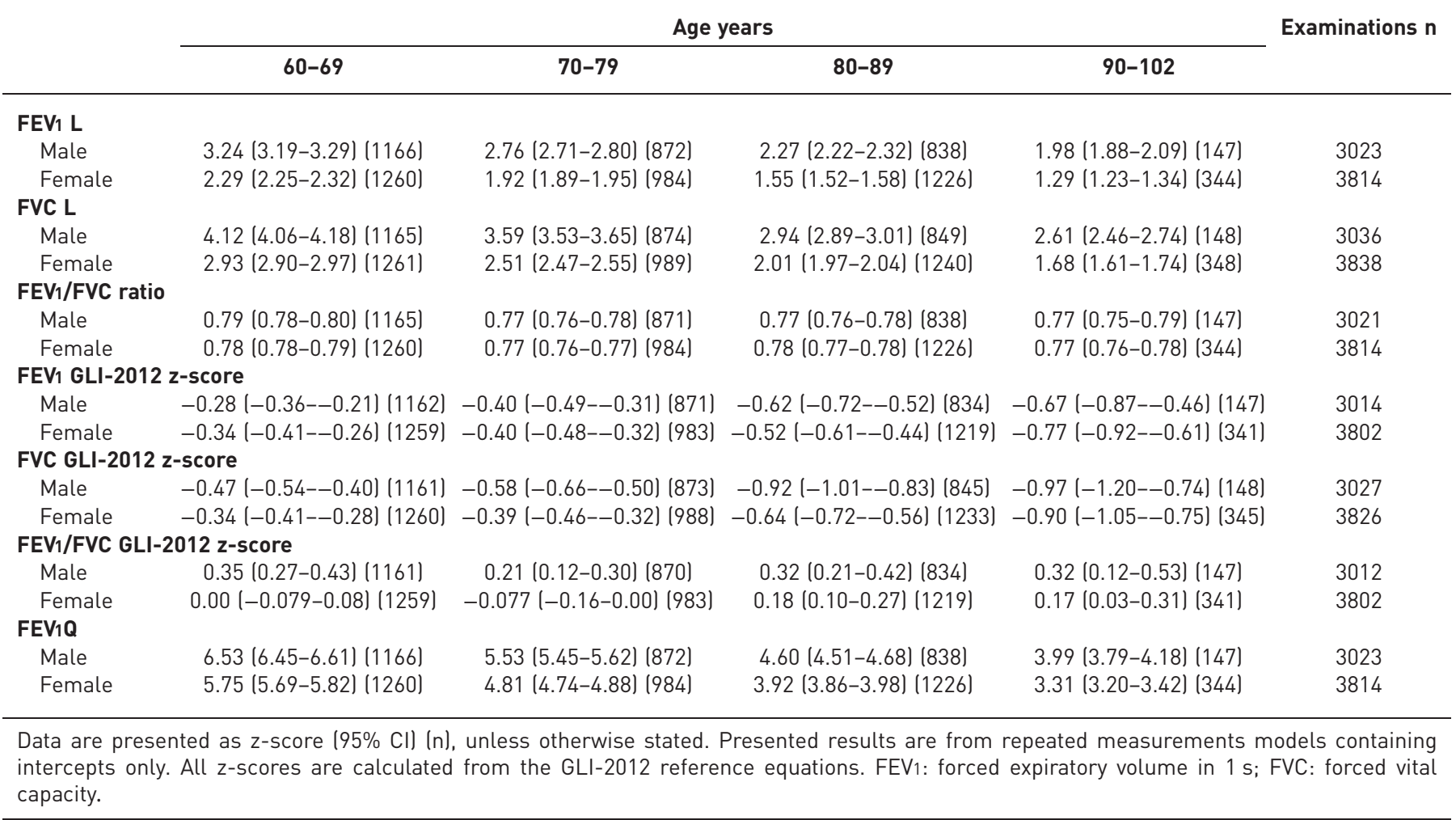

6.2\% for FEV1 and $8.8 \%$ for FVC. The proportions below the NHANES III LLN were 2.3\% for the FEV1/ FVC ratio, $2.3 \%$ for FEV1 and $2.1 \%$ for FVC.

The risk (OR, 95\% CI) of contributing with fewer than two spirometric measurements was significantly increased by markers of morbidity and frailty: active smoking habits relative to never-smokers $(1.6,1.4-1.9)$, being $>90$ years old relative to being $60-70$ years old $(2.7,2.0-3.7)$, longer time to walk $15 \mathrm{~m}(1.1,1.1-1.1$ per $1 \mathrm{~s}), \mathrm{FEV} 1$ and FVC below LLN $(5.4,4.7-6.1$ and 5.1, 4.4-5.8) and MMSE score <24 (4.1, 3.4-4.9).

\section{FEV1 and FVC change}

Absolute and relative FEV1 change are presented in tables 3 and 4 and supplementary tables S1 and S2 describe FVC change. Adjusted absolute change per year was $-56.2(-73.6--38.8) \mathrm{mL}$ for FVC. Adjusted relative change per year was $-2.46(-3.07--1.85) \%$ for FVC. Presented effects exclusively come from the multivariate analyses, unless otherwise stated. The significant risk factors for increased relative FVC and FEV1 decline were female sex, all age groups (compared to the youngest), being a current smoker (but only compared to ex-smokers for FVC), elevated CRP (only close to significant for FEV1, p=0.057) and low educational level. For increased absolute decline the risk factors were male sex, being a current smoker (compared to ex-smokers) for FEV1 and low education versus intermediate or high education for FVC. BMI $>35 \mathrm{~kg} \cdot \mathrm{m}^{-2}$ compared to BMI $20-25 \mathrm{~kg} \cdot \mathrm{m}^{-2}$ for decreased absolute decline in FEV 1 . One unit increase in CRP $\left(\mathrm{mg} \cdot \mathrm{L}^{-1}\right)$ increased relative FEV1 and FVC decline by 0.02 percentage points per year $(\mathrm{p}=0.019$ for $\mathrm{FVC})$, but did not significantly increase absolute decline $(-0.06 \mathrm{~mL}$ per year, $\mathrm{p}=0.74)$. Level of alcohol consumption did not increase relative or absolute FEV1 or FVC decline (results not shown). Adjusted and crude change per year for an ideal population stratified by risk factors are displayed in tables 3, 4, S1 and S2. The age effect on FEV1 and FVC change is illustrated in figure 1.

\section{Functional status}

Several markers of functional status correlated significantly to relative lung function decline, whereas absolute decline did not correlate to any marker (results not shown). When added to the full model both walking speed and TUG significantly influenced relative FEV1 change rates $(\mathrm{p}=0.0036$ and $\mathrm{p}=0.0056)$. TUG $(\mathrm{p}=0.0090)$, but not walking speed $(\mathrm{p}=0.30)$ significantly affected relative FVC change rate. There was no significant effect of 
TABLE 3 Individually calculated absolute forced expiratory volume in $1 \mathrm{~s}\left(F E V_{1}\right)$ change

Baseline FEV 1 L Crude FEV 1 absolute decline $\mathrm{mL}$ per year $(95 \% \mathrm{Cl})$
Adjusted (LSM) FEV1 absolute change

Trajectories

\begin{tabular}{|c|c|c|}
\hline & p-value & Full model $\mathrm{mL}$ \\
\hline
\end{tabular}

\begin{tabular}{|c|c|c|}
\hline \multicolumn{3}{|l|}{ Sex } \\
\hline Male & $3.09 \pm 0.69$ & $-49.1(-53.0--45.1)$ \\
\hline Female & $2.13 \pm 0.53$ & $-38.3(-41.9--34.7)$ \\
\hline \multicolumn{3}{|l|}{ Age years } \\
\hline $60-69$ & $2.83 \pm 0.74$ & $-41.7(-45.3--38.0)$ \\
\hline $70-79$ & $2.48 \pm 0.71$ & $-46.9(-52.7--41.1)$ \\
\hline $80-89$ & $2.12 \pm 0.62$ & $-42.9(-48.8--37.0)$ \\
\hline $90-102$ & $1.74 \pm 0.55$ & $-46.5(-60.8--32.2)$ \\
\hline \multicolumn{3}{|l|}{ Smoking habits } \\
\hline Current smoker & $2.45 \pm 0.75$ & $-47.0(-54.4--39.5)$ \\
\hline Ex-smoker & $2.67 \pm 0.75$ & $-41.2(-45.5--36.8)$ \\
\hline Never-smoker & $2.50 \pm 0.80$ & $-44.0(-48.4--39.7)$ \\
\hline \multicolumn{3}{|l|}{ Hypertension } \\
\hline Yes & $2.44 \pm 0.75$ & $-44.4 \mid--49.4--39.3$ \\
\hline No & $2.62 \pm 0.78$ & $-42.8(-46.0--39.5)$ \\
\hline \multicolumn{3}{|l|}{ Diabetes type II } \\
\hline Yes & $2.48 \pm 0.75$ & $-44.3(-55.9--32.8)$ \\
\hline No & $2.58 \pm 0.78$ & $-43.2(-46.2--40.2)$ \\
\hline \multicolumn{3}{|l|}{ CHD } \\
\hline Yes & $2.38 \pm 0.75$ & $-42.4(-50.2--34.6)$ \\
\hline No & $2.60 \pm 0.78$ & $-43.4(-46.3--40.4)$ \\
\hline \multicolumn{3}{|l|}{ Heart failure } \\
\hline Yes & $2.01 \pm 0.57$ & $-40.5(-54.9--26.1)$ \\
\hline No & $2.59 \pm 0.77$ & $-43.4(-46.3--40.4)$ \\
\hline \multicolumn{3}{|l|}{ BMI $\mathbf{k g} \cdot \mathbf{m}^{-2}$} \\
\hline$<20$ & $2.28 \pm 0.79$ & $-37.5(-57.8--17.3)$ \\
\hline $20-24.99$ & $2.58 \pm 0.80$ & $-46.4(-51.3--41.5)$ \\
\hline $25-35$ & $2.58 \pm 0.77$ & $-42.8(-46.3--39.3)$ \\
\hline$>35$ & $2.47 \pm 0.66$ & $-32.0(-44.0--20.1)$ \\
\hline \multicolumn{3}{|l|}{ Occupation } \\
\hline Low risk & $2.56 \pm 0.76$ & $-42.7(-45.7--39.7)$ \\
\hline Farming etc. & $2.34 \pm 0.74$ & $-42.3(-57.9--26.7)$ \\
\hline Unqualified work & $2.09 \pm 0.69$ & $-37.0(-49.3--24.8)$ \\
\hline Construction etc. & $2.96 \pm 0.74$ & $-50.3(-58.7--41.9)$ \\
\hline \multicolumn{3}{|l|}{ Education } \\
\hline Low & $2.18 \pm 0.66$ & $-46.4(-62.1--30.7)$ \\
\hline Intermediate & $2.51 \pm 0.77$ & $-41.3(-44.5--38.1)$ \\
\hline High & $2.78 \pm 0.76$ & $-48.2(-53.4--42.9)$ \\
\hline All & $2.57 \pm 0.78$ & $-43.2(-45.9--40.6)$ \\
\hline
\end{tabular}

$-51.7(-57.0--41.9)$
$-40.9(-45.8--36.1)$
$-42.8(-46.3--39.2)$
$-48.4(-54.2--42.6)$
$-44.7(-50.7--38.7)$
$-49.4(-63.7--35.3)$
$-50.3(-58.0--42.6)$
$-42.2(-47.4--37.0)$
$-46.4(-51.2--41.7)$

$<0.0001$
0.10
0.57
0.37

0.039
0.32

$-55.2(-67.5--42.9)$

$-48.3(-60.6--35.9)$

0.018

715

$-47.6(-59.8--35.3)$

$-54.3(-67.1--41.6)$

$-49.7(-62.2--37.2)$

$-55.4(-72.9--37.8)$

0.061

0.57

$-56.9(-70.2--43.4)$

$-47.9(-60.2--35.5)$

$-50.5(-63.0--37.9)$

0.03

0.13

$-53.7(-66--41.4)$

$-49.8(-62.2--37.3)$

0.20

$-51.0(-66.1--35.9)$

$-52.5(-63.5--41.4)$

0.80

$-51.2(-64.3--38.1)$

$-52.2(-64.6--39.9)$

0.8

$-52.7(-68.5--36.9)$

$-50.8(-62.1--39.6)$

0.79

$-54.1(-78.9--29.2)$

$-57.1(-68.9--45.2)$

$-51.4(-62.3--40.4)$

$-44.4(-58.5--30.3)$

0.06

0.041

$-49.7(-61.1--38.3)$

$-50.1(-68.6--31.6)$

$-49.3(-64.9--33.7)$

$-57.8(-71.9--43.7)$

0.96

0.96

0.08

$-57.8(-76.5--33.9)$

$-44.9(-55.8--34.0)$

$-52.4(-64.9--40.0)$

$-51.7(-63.7--39.8)$
0.29

844

839

339

325

56

224

661

674

452

1107

98

1461

195

1364

65

1494

29

491

956

83

1280

46

75

158

46

$0.12 \quad 1103$

$0.53 \quad 410$

1559

Data are presented as mean \pm SD or $n$, unless otherwise stated. Basic model is adjusted for the categorical variables age, sex and smoking status. The full model is additionally adjusted for the categorical variables hypertension, coronary heart disease (CHD), heart failure, diabetes type II, occupation, body mass index (BMI), education and the continuous variables C-reactive protein and alcohol consumption. Values used for least squares means (LSM) calculations were alcohol consumption 8.4 glasses per month and CRP $5.4 \mathrm{mg} \cdot \mathrm{L}^{-1}$. Missing values for covariates resulted in the exclusion of 25 trajectories.

grip strength on either relative FEV1 $(\mathrm{p}=0.061)$ or FVC change $(\mathrm{p}=0.24)$. In addition, walking speed, grip strength and TUG were highly correlated to FEV1 and FVC GLI-2012 z-scores in the total population $(\mathrm{p}<0.0005$ for all) with the exception of the combination FVC GLI-2012 z-score and TUG ( $\mathrm{p}=0.083)$.

\section{Sensitivity analysis}

Pairs of spirometries were of two types. Either a bronchodilator was given only at the second measurement (type 1) or at both measurements (type 2). Type 1 corresponded to a lower adjusted overall rate of relative decline in FEV1 and FVC compared to type 2 ( 0.0019 per year and 0.005 per year, corresponding to a difference in relative decline per year of $0.19 \%$ and $0.5 \%$, respectively). However, these differences were not statistically significant $\left(\mathrm{p}=0.38\right.$ for $\mathrm{FEV}_{1}$ and $\mathrm{p}=0.26$ for $\left.\mathrm{FVC}\right)$. In the absolute analysis, type 1 corresponded to a slightly higher rate of FEV1 decline $(3 \mathrm{~mL}$ per year) and a lower rate of FVC decline 
TABLE 4 Individually calculated relative forced expiratory volume in $1 \mathrm{~s}$ (FEV1) change

Baseline FEV1 L Crude FEV1 relative change \%

Adjusted (LSM) FEV1 relative change

Trajectories per year $(95 \% \mathrm{CI})$

\begin{tabular}{|c|c|c|}
\hline $\begin{array}{l}\text { Basic model } \% \\
\text { per year }(95 \% \mathrm{Cl})\end{array}$ & p-value & $\begin{array}{l}\text { Full model \% } \\
\text { per year }(95 \% \mathrm{Cl})\end{array}$ \\
\hline
\end{tabular}

\begin{tabular}{|c|c|c|}
\hline \multicolumn{3}{|l|}{ Sex } \\
\hline Male & $3.09 \pm 0.69$ & $-1.80(-1.99--1.61)$ \\
\hline Female & $2.13 \pm 0.53$ & $-2.07(-2.24--1.89)$ \\
\hline \multicolumn{3}{|l|}{ Age years } \\
\hline $60-69$ & $2.83 \pm 0.74$ & $-1.64(-1.82--1.47)$ \\
\hline $70-79$ & $2.48 \pm 0.71$ & $-2.14(-2.41--1.86)$ \\
\hline $80-89$ & $2.12 \pm 0.62$ & $-2.35(-2.64--2.07)$ \\
\hline $90-102$ & $1.74 \pm 0.55$ & $-3.00(-3.67--2.32)$ \\
\hline \multicolumn{3}{|l|}{ Smoking habits } \\
\hline Current smoker & $2.45 \pm 0.75$ & $-2.23(-2.59--1.87)$ \\
\hline Ex-smoker & $2.67 \pm 0.75$ & $-1.79(-2.00--1.58)$ \\
\hline Never-smoker & $2.50 \pm 0.80$ & $-2.00(-2.21--1.80)$ \\
\hline \multicolumn{3}{|l|}{ Hypertension } \\
\hline Yes & $2.44 \pm 0.75$ & $-2.06(-2.30--1.81)$ \\
\hline No & $2.62 \pm 0.78$ & $-1.90(-2.06--1.75)$ \\
\hline \multicolumn{3}{|l|}{ Diabetes type II } \\
\hline Yes & $2.48 \pm 0.75$ & $-2.02(-2.57--1.46)$ \\
\hline No & $2.58 \pm 0.78$ & $-1.94(-2.09--1.80)$ \\
\hline \multicolumn{3}{|l|}{ CHD } \\
\hline Yes & $2.38 \pm 0.75$ & $-2.11(-2.48--1.73)$ \\
\hline No & $2.60 \pm 0.78$ & $-1.92(-2.07--1.78)$ \\
\hline \multicolumn{3}{|l|}{ Heart failure } \\
\hline Yes & $2.01 \pm 0.57$ & $-2.35(-3.04--1.65)$ \\
\hline No & $2.59 \pm 0.77$ & $-1.93(-2.07--1.78)$ \\
\hline \multicolumn{3}{|l|}{ BMI $\mathbf{k g} \cdot \mathbf{m}^{-2}$} \\
\hline$<20$ & $2.28 \pm 0.79$ & $-1.88(-2.86--0.89)$ \\
\hline $20-24.99$ & $2.58 \pm 0.80$ & $-2.03(-2.26--1.79)$ \\
\hline $25-35$ & $2.58 \pm 0.77$ & $-1.94(-2.11--1.77)$ \\
\hline$>35$ & $2.47 \pm 0.66$ & $-1.54(-2.12--0.96)$ \\
\hline \multicolumn{3}{|l|}{ Occupation } \\
\hline Low risk & $2.56 \pm 0.76$ & $-1.93(-2.07--1.79)$ \\
\hline Farming etc. & $2.34 \pm 0.74$ & $-1.88(-2.63--1.12)$ \\
\hline Unqualified work & $2.09 \pm 0.69$ & $-2.18(-2.77--1.58)$ \\
\hline Construction etc. & $2.96 \pm 0.74$ & $-1.90(-2.31--1.49)$ \\
\hline \multicolumn{3}{|l|}{ Education } \\
\hline Low & $2.18 \pm 0.66$ & $-2.48(-3.23--1.73)$ \\
\hline Intermediate & $2.51 \pm 0.77$ & $-1.91(-2.06--1.75)$ \\
\hline High & $2.78 \pm 0.76$ & $-1.99 \mid-2.24--1.73$ \\
\hline All & $2.57 \pm 0.78$ & $-1.94 \mid-2.08--1.8$ \\
\hline
\end{tabular}

$-2.28(-2.53-2.02)$

$-2.51(-2.75--2.28)$

$-1.74(-1.91--1.57)$

$-2.26(-2.53--1.98)$

$-2.47(-2.76--2.18)<0.0001$

$-3.10(-3.78--2.42) \quad 0.0001$

$-2.18(-2.43--1.93)$

$-2.23(-2.46--2.00)$

0.0015
0.0038
$-2.78(-3.15--2.41)$

$-276(-3.34--2.18)$

$-3.18(-3.76--2.60) \quad 0.0020$

$-2.31(-2.89--1.73)$

$-2.90(-3.50--2.30)$

$-2.98(-3.57--2.39)$

$-3.68(-4.50--2.85)$

$-3.41(-4.03--2.78)$

$-2.76(-2.34--2.17)$

$-2.74(-3.33--2.15)$

$-3.04(-3.62--2.46)$

$-2.90(-3.48--2.31)$

$-3.02(-3.73--2.31)$

$-2.92(-3.48--2.31)$

$-3.01(-3.63--2.39)$

$-2.93(-3.51--2.35)$

$-3.11(-3.85--2.37)$

$-2.83(-3.36--2.29)$

$-3.09(-4.25--1.92)$

$-3.11(-3.67--2.55)$

$-2.99(-3.51--2.47)$

$-2.68(-3.35--2.01)$

$-2.86(-3.40--2.32)$

$-2.87(-3.73--1.99)$

$-2.95(-3.68--2.22)$

$-3.20(-3.86--2.54)$

$-3.51(-4.38--2.62)$

$-2.56(-3.07--2.04)$

$-2.85(-3.43--2.26)$

$-2.39(-2.61--2.18)$

$2.97(-3.53--2.40)$
715

844

839

339

325

56

224

661

674

452

1107

98

1461

195

$0.69 \quad 1364$

65

$0.37 \quad 1494$

$0.97 \quad 29$

491

$0.14 \quad 956$

1280

$0.99 \quad 46$

$0.76 \quad 75$

$0.12 \quad 158$

46

$0.0144 \quad 1103$

$0.10 \quad 410$

Data are presented as mean \pm SD or $n$, unless otherwise stated. Basic model is adjusted for the categorical variables age, sex and smoking status. The full model is additionally adjusted for the categorical variables hypertension, coronary heart disease (CHD), heart failure, diabetes type II, occupation, body mass index (BMI), education and the continuous variables C-reactive protein and alcohol consumption. Values used for least squares means (LSM) calculations were alcohol consumption 8.4 glasses per month and CRP $5.4 \mathrm{mg} \cdot \mathrm{L}^{-1}$. Missing values for covariates resulted in the exclusion of 25 trajectories.

( $8 \mathrm{~mL}$ per year). Differences were not statistically significant ( $\mathrm{p}=0.59$ and 0.33 , respectively). Effects of risk factors did not significantly differ between the two types for any outcome.

\section{Discussion}

Brief synopsis of key findings

In the relative analysis, we confirmed the hypothesis that age, female sex, current smoking habits, low educational level and elevated CRP increased FEV1 and FVC decline. None of these, except smoking, are widely accepted risk factors for rate of lung function decline. In the absolute analysis, current (compared to former) smoking was confirmed as a risk factor for increased FEV1 and low educational level for FVC decline, while BMI $>35 \mathrm{~kg} \cdot \mathrm{m}^{-2}$ significantly decreased decline for FEV 1 . For absolute decline, male sex rather than female sex increased the rate of decline. 

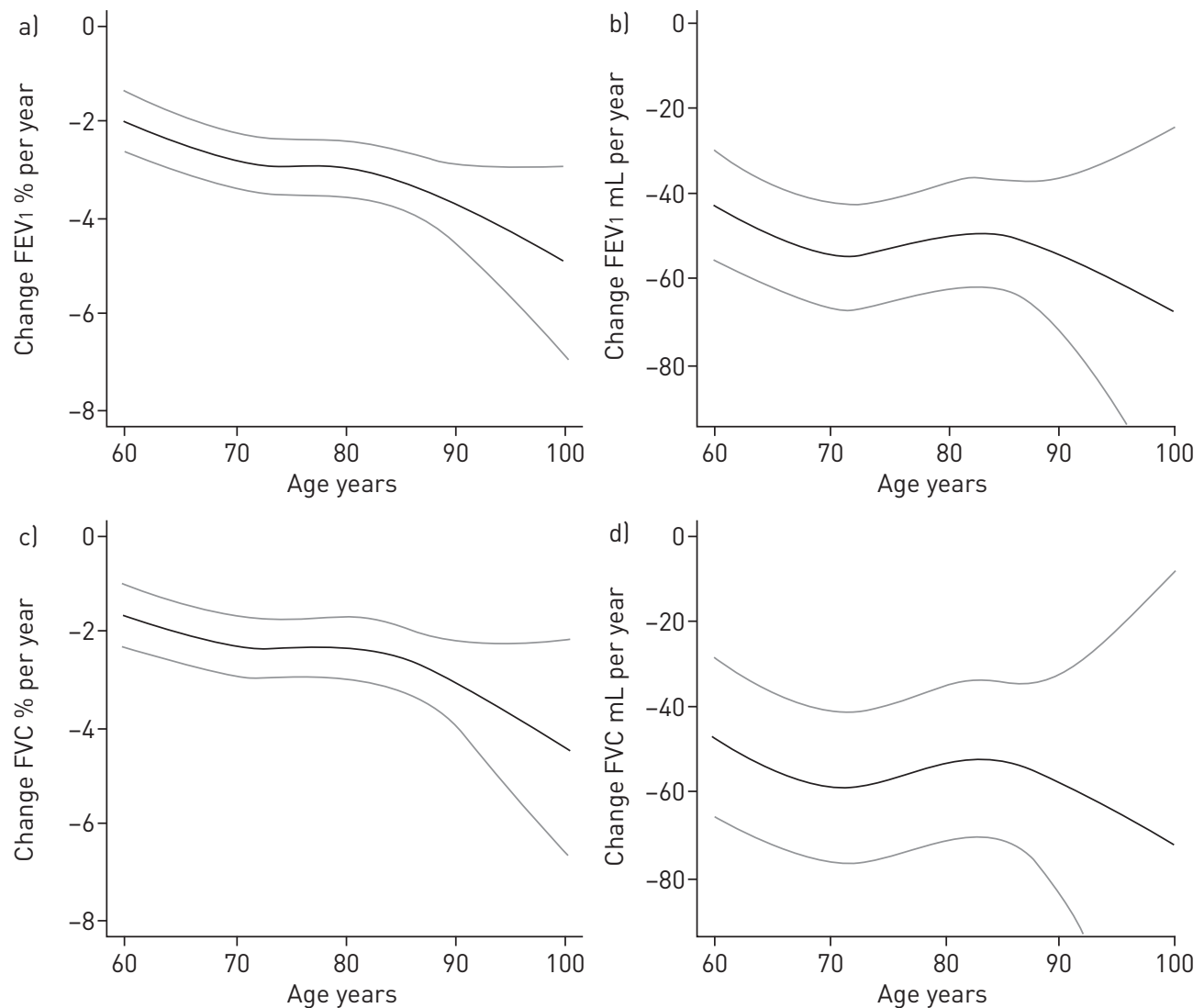

FIGURE 1 The a) relative and $b$ ) absolute change in forced expiratory volume in $1 \mathrm{~s}$ (FEV 1 ) and c) relative and d) absolute change in forced vital capacity (FVC) for ages 60-102 years. Adjusted for the categorical variables sex, smoking habits, heart failure, hypertension, diabetes, coronary heart disease, socioeconomic status and occupation and the continuous variables alcohol consumption and C-reactive protein. Age was modelled as a continuous variable using restricted cubic splines.

\section{Interpretation and comparison with previous studies \\ Relative and absolute change}

Refer to the statistical appendix in the supplementary material for more background on relative and absolute change. We found some support in our data for the relevance of relative change in a risk factors analysis. In a separate analysis presented in the supplementary material we discovered that absolute but not relative change was significantly associated to initial volume, giving some credit to the theory that change is relative in nature. This will lead to bias if it is not accounted for [16]. To exemplify the importance of initial volume, for FEV1 change we would expect that current smokers with the same initial lung volumes lose a larger absolute volume per time unit compared to never-smokers. However, in this study the starting volume is lower for the never-smokers and the difference in absolute decline is expected to be reduced. This is what we find in this case, leading to a nonsignificant difference in decline between current smokers and never-smokers for absolute decline. For relative change we do not have this bias and as expected current smoking increases relative decline compared to both ex-smokers and never-smokers. To compare the clinical relevance of relative and absolute change we correlated them with markers of functional status. Relative change was significantly related to several clinically relevant functional markers, but absolute change was not, suggesting a weak correlation between absolute change and well established functional parameters. For more details on this analysis and on the models of change, refer to the statistical section in the supplementary material. We request more well designed studies on lung function change in the elderly with focus on the nature of change (shape of the decline) and its relationship to clinically relevant outcomes such as death.

Age

Previous studies that report lung function change values in general elderly populations differ methodologically and do not report findings for ages $\geqslant 90$ years, but they can still be used for comparison $[1,4,34]$. One of these studies measured change relatively [1] and the other studies modelled absolute 
levels of lung function using linear mixed effects regression models and assessed change through suitable adjustment. Two of these studies reported weak or no age effect on rate of FEV1 decline or risk of being a "rapid decliner" in FEV1 [1,4]. An increased decline in FEV1 with higher age was reported by VAZ FrAGoso et al. [34] and in a Korean COPD population [35]. Theories on normal and pathological ageing of the lung include frequent/inadequate response to respiratory infections, oxidative stress and cumulative exposure to toxins leading to dysregulated inflammation [36]. Additionally, physiological changes with loss of elastic recoil, weakening of respiratory muscles and increased rigidity of the chest wall contribute [37].

\section{FEV1Q}

The cross-sectional reduction in FEV1Q was about one turnover per decade, which is the same as previous estimates for smokers aged $>60$ years [32]. That the reduction by age of FEV1Q was not lower than an all-smoking population could possibly be explained by the high age of the population in the current study, or other differences between populations [32]. The ratio of absolute decline in FEV1 between females and males for this study was $\sim 0.8$, which is similar to previous findings [32]. This is the exact ratio between the FEV1 values of males and females at the 1st percentile that the FEV1Q formula is based upon. One interpretation of this is that the longitudinal overall reductions in FEV1Q by age can be broadly applied to both sexes [32]. This may be useful since FEV1Q has been suggested as a staging tool for COPD, and forecasting future levels may be of clinical use [38].

Sex

A sex-specific causal relationship with lung function decline is yet to be established $[3,8,39,40]$. Female sex was linked to increased decline of FEV1 in two out of the three relevant studies that reported sex-specific estimates. One of these studies measured change relatively while the other differed methodologically, making direct comparison difficult [1,4]. It has previously been demonstrated that female COPD subjects had greater relative but not absolute decline in FEV1 [41]. The sex effect observed in this study on relative lung function decline is not likely to be explained by socioeconomic differences between sexes (including smoking habits) or a higher proportion of females in the oldest age groups, since these factors have been adjusted for. Furthermore, it is not likely to be due to systemic inflammation, since the sex effect remains after adjusting for CRP. One explanation for this finding is that females may be more sensitive to tobacco smoke, suggesting an interaction between sex and smoking habits [5, 10]. However, this interaction was nonsignificant when added to the full model for both FEV1 and FVC decline. Other previous theories on the female sex effect include sex hormones [39], dimensional differences [40] and sex-specific differences in the morphology of COPD [42].

\section{CRP}

Elevated CRP levels have been linked previously to increased FEV1 decline in elderly general populations [4]. There is ongoing research on potential biomarkers for COPD disease activity and the role of CRP in this context is still unclear $[5,13]$. It has been suggested that the association between high CRP and poor lung function may be the result of an age-related inflammation nicknamed "inflammaging" [26]. However, a strong age effect remains in this study after adjusting for CRP and we did not find any significant interaction between CRP and age groups for either FEV1 or FVC decline. In other words, the effect of systemic inflammation on lung function decline seems to be constant across ages.

\section{Education and socioeconomic status}

Even after adjusting for smoking habits, sex, alcohol consumption, coronary heart disease and occupational exposure, subjects reporting a low level of attained education had a greater rate of yearly FEV1 and FVC decline (except absolute decline of FEV1). Effects of educational level on lung function have been described before, but longitudinal studies are rare and have often not been adjusted for possible confounders such as occupation and alcohol consumption $[1,4,9]$. However, the effects of education did not remain when using robust regression procedures, and should be interpreted with caution. Our data do not clearly tell whether the results are due to chance and a small number of participants with low educational level or true differences in patterns of changes of lung volumes. The low educational level group seems to represent a high level of heterogeneity of change of lung volumes, which may reflect that effects of a low socioeconomic status are complex and differ greatly between individuals. The effects of low education may enter early in life: low intrauterine growth, childhood respiratory tract infections and poor nutrition and housing have all been suggested [9]. Low education level $(<12$ years/no degree) was close to significant compared to high education level (>12 years/degree) in one of the studies [1] on general elderly populations previously mentioned, but not in the other study that tested for education level as a predictor [4]. These categories of education level are roughly, but not directly, comparable to this study's categorisation due to differences in educational systems. 


\section{Comorbidities}

A $\mathrm{BMI}>35 \mathrm{~kg} \cdot \mathrm{m}^{-2}$ decreased absolute FEV1 decline. Apart from this, we did not find that comorbidities or BMI significantly correlated to FEV1 or FVC decline. Previous studies have found associations between lung function and coronary heart disease [6], heart failure [24], the metabolic syndrome and high BMI $[25,27,28]$. That $\mathrm{BMI}>35 \mathrm{~kg} \cdot \mathrm{m}^{-2}$ reduced absolute $\mathrm{FEV} 1$ decline may be due to change being relative in nature and obesity leading to reduced lung volumes, which in turn leads to smaller subsequent absolute decline values $[25,27,28]$. This supports the idea that relative change is preferable to absolute change in a risk factor analysis. Most of these previous studies have been cross-sectional in design where reversed causality may be a concern. There are no previous studies accounting for socioeconomics, occupation and CRP. This may have confounded the results.

\section{Limitations}

Longitudinal studies on very old participants are often subject to dropout due to morbidity or death of participants. We have addressed this by making house calls, conducting examinations in nursing homes and by examining those aged $\geqslant 80$ years more frequently. Still, after adjusting for age and sex, lower functional status, having an FEV1 and FVC GLI-2012 z-score below the LLN and being a smoker was significantly associated with failure to complete more than two examinations with an acceptable spirogram. Functional status was correlated to FEV1 and FVC GLI-2012 z-scores and relative change rates. $39 \%$ of those who failed to complete any spirometry at all had a MMSE score <24, which is likely to reflect the inability to perform a technically acceptable spirometry with a cognitive disorder [42]. To further reduce selection bias among the old and frail, we included a handful of subjects $(1.9 \%$ of all spirometries, $5.1 \%$ of those aged $\geqslant 90$ years) with only one acceptable manoeuvre. This is in contrast to younger subjects who should be able to perform multiple acceptable manoeuvres or when spirometry is used diagnostically in a clinical context $[20,23]$. In spite of this we probably underestimate the true reduction in lung function by selection of the fittest. MANNino et al. [1] reported a dropout of $60 \%$ among those aged $\geqslant 86$ years, which was very close to this study's percentage of participants with only one spirometry measurement $(62 \%)$ in the same age group.

At baseline, cohort II participants were on average 7.5 years younger than cohort I. This is because for cohort I subjects were aged 60-95 years at recruitment and cohort II subjects were aged 60-81 years. That is, those who received a bronchodilator at baseline were somewhat younger than those who did not, possibly leading to an underestimation of the observed age effect. However, adjusted decline rates and effects of risk factors did not significantly differ between data pairs with bronchodilation at first measurement and those without. Otherwise, cohort I and cohort II were examined using the same equipment, the same staff and were both randomly selected from the same population with similar participation rates. Bronchodilators may cause adverse reactions among elderly, which could affect spirometry performance and participation [43]. Studies have shown that relative bronchodilator response may be greater with lower FVC or FEV1 values, which could introduce age-related differences in bronchodilator response [2].

Farmers and construction workers had elevated levels of FEV1 decline compared to low-risk occupations, but these effects did not reach statistical significance. It is likely that with more data the occupational categories could be further subcategorised to identify specific subgroups that would be more exposed to dust and fumes. We did not have access to self-reported exposure to dust and fumes, which would have been helpful in distinguishing the risk groups from the reference groups [44]. Moreover, this is a study of mostly retired subjects, thus only examining long-term effects of previous occupational exposures. Considering the large difference between current and former exposure to tobacco smoke found in this study, we should not conclude that our findings can be generalised to current occupational exposure.

\section{NHANES III may offer a better choice for elderly subjects}

There is uncertainty regarding reference equations in very high ages and although GLI-2012 includes ages up to 95 years, it is noteworthy that extrapolated values from NHANES III have been shown to correlate better with survival and are possibly more accurate at high ages [45]. In this study, NHANES III z-scores were generally closer to zero compared with GLI-2012 z-scores. Moreover, among never-smokers we found the proportion of NHANES III z-scores $<2.5 \%$ LLN to be $2.1-2.3 \%$ for FEV1, FVC and FEV1/FVC, but GLI-2012 z-scores were roughly two to four times higher, depending on index. This supports previous findings that NHANES III z-scores may offer a better choice for elderly populations. Presented GLI-2012 Z-scores for subjects aged $>95$ years are likely to be slightly too low, since age was set to 95 years for these subjects due to a lack of reference equations. 


\section{Generalisability}

This is a study on a randomly selected general population from cities and rural areas. There was no exclusion criterion other than not performing at least one acceptable spirometry. Additionally, the age spectrum with no upper age limit and ability to make house calls to those unable to visit the clinic due to frailty also contribute to the study's generalisability. However, age and morbidity associated dropouts may have led to bias by attrition; this is discussed further in the Limitations section. The baseline FEV1 GLI-2012 z-score for subjects with at least two spirometry measurements of -0.27 is comparable to the reported FEV1 z-score of -0.21 in another slightly younger general population, suggesting that they are similar in terms of normality of lung function [34].

\section{Conclusion and possible contributions of this study}

This is the first longitudinal study to specifically observe and analyse individual FEV 1 and FVC change in ages $>90$ years, thereby providing essential information on the natural history of lung function. Moreover, this study highlights the difference in outcome resulting from studying relative as opposed to absolute change. The rate of relative FEV1 and FVC decline increased with age, female sex, elevated CRP (only marginally significant for FEV1), current smoking habits and low educational level. The rate of absolute decline decreased with BMI $>35 \mathrm{~kg} \cdot \mathrm{m}^{-2}$ and increased with male sex and current smoking habits for FEV 1 and low education for FVC. In conclusion, risk factors for increased lung function decline will very much depend on how we model change. For a potential risk factor having a strong influence on initial volume (such as female sex) it is crucial to choose an appropriate model as outcomes of different models may be diametrically opposite. We found some support for the relevance of relative change when studying risk factors of lung function decline. In addition, we found that NHANES III may offer better reference equations for elderly compared to GLI-2012. These findings should be replicated and further investigated.

Acknowledgements: We would like to acknowledge the comprehensive feedback from the editors and reviewers during the development of this manuscript.

Conflict of interest: J. Luoto reports grants from Skåne County, during the conduct of the study. M. Pihlsgård has nothing to disclose. P. Wollmer reports grants from Swedish Heart and Lung Foundation, during the conduct of the study; personal fees for lecturing from AstraZeneca $\mathrm{AB}$, outside the submitted work; in addition, P. Wollmer has a patent device and method for pulmonary function measurement pending. S. Elmståhl reports grants from Swedish Research Council (grant numbers 521-2013-8604 and 2017-01613), during the conduct of the study.

Support statement: This study was funded by Skåne County (621061) and the Swedish Research Council (2017-01613, 521-2013-8604). Funding information for this article has been deposited with the Crossref Funder Registry.

\section{References}

Mannino DM, Davis KJ. Lung function decline and outcomes in an elderly population. Thorax 2006; 61: 472-477. Pellegrino R. Interpretative strategies for lung function tests. Eur Respir J 2005; 26: 948-968.

Global Initiative for Chronic Obstructive Lung Disease (GOLD). Global Strategy for the Diagnosis, Management and Prevention of COPD. 2014. Available from: http://goldcopd.org/.

4 Yohannes AM, Tampubolon G. Changes in lung function in older people from the English Longitudinal Study of Ageing. Expert Rev Respir Med 2014; 8: 515-521.

5 Vestbo J, Edwards LD, Scanlon PD, et al. Changes in forced expiratory volume in 1 second over time in COPD. N Engl J Med 2011; 365: 1184-1192.

6 Burchfiel CM, Marcus EB, Sharp DS, et al. Characteristics associated with rapid decline in forced expiratory volume. Ann Epidemiol 1996; 6: 217-227.

7 Fletcher C, Peto R. The natural history of chronic airflow obstruction. Br Med J 1977; 1: 1645-1648.

8 Luoto JA, Elmståhl S, Wollmer P, et al. Incidence of airflow limitation in subjects 65-100 years of age. Eur Respir J 2016; 47: 461-472.

9 Hegewald MJ, Crapo RO. Socioeconomic status and lung function. Chest 2007; 132: 1608-1614.

10 Krzyzanowski M, Jedrychowski W, Wysocki M. Factors associated with the change in ventilatory function and the development of chronic obstructive pulmonary disease in a 13-year follow-up of the Cracow Study: risk of chronic obstructive pulmonary disease. Am Rev Respir Dis 1986; 134: 1011-1019.

11 Mirabelli MC, London SJ, Charles LE, et al. Occupation and three-year incidence of respiratory symptoms and lung function decline: the ARIC Study. Respir Res 2012; 13: 24.

12 Humerfelt S, Gulsvik A, Skjaerven R, et al. Decline in FEV1 and airflow limitation related to occupational exposures in men of an urban community. Eur Respir J 1993; 6: 1095-1103.

13 Celli BR, Decramer M, Wedzicha JA, et al. An official American Thoracic Society/European Respiratory Society statement: research questions in COPD. Eur Respir J 2015; 45: 879-905.

14 Thomsen LH, Dirksen A, Shaker SB, et al. Analysis of FEV1 decline in relatively healthy heavy smokers: implications of expressing changes in FEV1 in relative terms. COPD 2014; 11: 96-104.

15 Vestbo J, Lange P. Fletcher and Peto 40 years on. A tribute and reflection. Am J Respir Crit Care Med 2017; 195: $1420-1422$.

16 Raimondi GA. FEV 1 decline in patients with chronic obstructive pulmonary disease. Am J Respir Crit Care Med 2017; 195: 1676-1677.

17 Ekström H, Elmståhl S. Pain and fractures are independently related to lower walking speed and grip strength: results from the population study "Good Ageing in Skåne.". Acta Orthop 2006; 77: 902-911. 
18 Stenhagen M, Ekström H, Nordell E, et al. Falls in the general elderly population: a 3- and 6-year prospective study of risk factors using data from the longitudinal population study 'Good ageing in Skane'. BMC Geriatr 2013; 13: 81 .

19 Lagergren M, Fratiglioni L, Hallberg IR, et al. A longitudinal study integrating population, care and social services data. The Swedish National Study on Aging and Care (SNAC). Aging Clin Exp Res 2004; 16: 158-168.

20 Standardization of spirometry, 1994 update. Am J Respir Crit Care Med 1995; 152: 1107-1136.

21 Nordenskiöld UM, Grimby G. Grip force in patients with rheumatoid arthritis and fibromyalgia and in healthy subjects. A study with the Grippit instrument. Scand J Rheumatol 1993; 22: 14-19.

22 Folstein MF, Folstein SE, McHugh PR. "Mini-mental state". A practical method for grading the cognitive state of patients for the clinician. J Psychiatr Res 1975; 12: 189-198.

23 Fragoso CA. Epidemiology of chronic obstructive pulmonary disease (COPD) in aging populations. COPD 2016; 13: $125-129$.

24 Lizak MK, Zakliczyński M, Jarosz A, et al. The influence of chronic heart failure on pulmonary function tests in patients undergoing orthotopic heart transplantation. Transplant Proc 2009; 41: 3194-3197.

25 Chen W-L, Wang C-C, Wu L-W, et al. Relationship between lung function and metabolic syndrome. PLoS One 2014; 9: e108989.

26 Ahmadi-Abhari S, Kaptoge S, Luben RN, et al. Longitudinal association of C-reactive protein and lung function over 13 years: the EPIC-Norfolk study. Am J Epidemiol 2014; 179: 48-56.

27 Wang S, Sun X, Hsia T-C, et al. The effects of body mass index on spirometry tests among adults in Xi'an, China Medicine 2017; 96: e6596.

28 Jones RL, Nzekwu M-MU. The effects of body mass index on lung volumes. Chest 2006; 130: 827-833.

29 Quanjer PH, Stanojevic S, Cole TJ, et al. Multi-ethnic reference values for spirometry for the 3-95-yr age range: the global lung function 2012 equations. Eur Respir J 2012; 40: 1324-1343.

30 Hankinson JL, Odencrantz JR, Fedan KB. Spirometric reference values from a sample of the general U.S. population. Am J Respir Crit Care Med 1999; 159: 179-187.

31 Stanojevic S. GLI-2012 SAS Macro Version 2. www.ers-education.org/guidelines/global-lung-function-initiative/ spirometry-tools/sas-macro.aspx Date last updated: April 7, 2013.

32 Miller MR, Pedersen OF. New concepts for expressing forced expiratory volume in $1 \mathrm{~s}$ arising from survival analysis. Eur Respir J 2010; 35: 873-882.

33 Quanjer PH, Stanojevic S, Cole TJ, et al. Implementing GLI 2012 lung function regression equations. www. lungfunction.org/files/implementingGLIequations.pdf Date last accessed: June 15, 2015.

34 Vaz Fragoso CA, McAvay G, Van Ness PH, et al. Aging-related considerations when evaluating the forced expiratory volume in 1 second (FEV1) over time. J Gerontol A Biol Sci Med Sci 2016; 71: 929-934.

35 Kim SJ, Lee J, Park YS, et al. Age-related annual decline of lung function in patients with COPD. Int J Chron Obstruct Pulmon Dis 2015; 11: 51-60.

36 Vaz Fragoso CA, Lee PJ. The aging lung. J Gerontol A Biol Sci Med Sci 2012; 67: 233-235.

37 Vaz Fragoso CA, Gill TM. Respiratory impairment and the aging lung: a novel paradigm for assessing pulmonary function. J Gerontol A Biol Sci Med Sci 2012; 67: 264-275.

38 Culver BH. Assessment of severity and prognosis in COPD: moving beyond percent of predicted. Eur Respir $J$ 2018; 52: 1801005

39 Becklake MR, Kauffmann F. Gender differences in airway behaviour over the human life span. Thorax 1999; 54: $1119-1138$.

40 Kanner RE, Connett JE, Altose MD, et al. Gender difference in airway hyperresponsiveness in smokers with mild COPD. The Lung Health Study. Am I Respir Crit Care Med 1994; 150: 956-961.

41 Celli BR, Thomas NE, Anderson JA, et al. Effect of pharmacotherapy on rate of decline of lung function in chronic obstructive pulmonary disease: results from the TORCH study. Am J Respir Crit Care Med 2008; 178: 332-338.

42 Dransfield MT, Washko GR, Foreman MG. Gender differences in the severity of CT emphysema in COPD. Chest 2007; 132: 464-470.

43 Gershon A, Croxford R, Calzavara A, et al. Cardiovascular safety of inhaled long-acting bronchodilators in individuals with chronic obstructive pulmonary disease. JAMA Intern Med 2013; 173: 1175-1185.

44 Trupin L, Earnest G, San Pedro M, et al. The occupational burden of chronic obstructive pulmonary disease. Eur Respir J 2003; 22: 462-469.

45 Allen SC, Yeung P. Inability to draw intersecting pentagons as a predictor of unsatisfactory spirometry technique in elderly hospital inpatients. Age Ageing 2006; 35: 304-306. 